\title{
Six-dimensional regularization of chiral gauge theories on a lattice*
}

\section{Hidenori Fukaya ${ }^{\dagger}$, Tetsuya Onogi, Shota Yamamoto, and Ryo Yamamura ${ }^{\dagger}$}

Department of Physics, Osaka University, Toyonaka, Osaka 560-0043 Japan

\begin{abstract}
We propose a six-dimensional regularization of four dimensional chiral gauge theories. We consider a massive Dirac fermion in six dimensions with two different operators having domain-wall profiles in the fifth and the sixth directions, respectively. A Weyl fermion appears as a localized mode at the junction of the two domain-walls. In our formulation, the Stora-Zumino chain of the anomaly descent equations, starting from the axial $U(1)$ anomaly in six-dimensions to the gauge anomaly in four-dimensions, is naturally embedded. Moreover, a similar inflow of the global anomalies is found. The anomaly free condition is equivalent to requiring that the axial $U(1)$ anomaly and the parity anomaly are canceled among the six-dimensional Dirac fermions. Putting the gauge field at the four- dimensional junction and extending it to the bulk using the Yang-Mills gradient flow, as recently proposed by Grabowska and Kaplan, we define the four-dimensional path integral of the target chiral gauge theory.
\end{abstract}

34th annual International Symposium on Lattice Field Theory

24-30 July 2016

University of Southampton, $U K$

${ }^{*}$ This is a joint contribution for two talks "Six-dimensional regularization of chiral gauge theories on a lattice (I \& II)" given by two of the authors.

${ }^{\dagger}$ Speakers. 


\section{Introduction}

In our textbooks on quantun field theory [四, we learn that the consistent form of gauge anomaly of the Weyl fermions is obtained from the axial $U(1)$ anomaly in 6-dimensions via 5dimensional parity anomaly:

$$
\begin{aligned}
\operatorname{Tr}\left[F^{3}\right]= & d \operatorname{Tr}\left[A(d A)^{2}+\frac{3}{2} A^{3} d A+\frac{3}{5} A^{5}\right], \\
& \delta_{v} \operatorname{Tr}\left[A(d A)^{2}+\frac{3}{2} A^{3} d A+\frac{3}{5} A^{5}\right]=d\left\{v^{a} \operatorname{Tr}\left[T^{a} d\left(A d A+\frac{1}{2} A^{3}\right)\right]\right\} .
\end{aligned}
$$

Here we have used the differential form for the gauge field $A=A_{\mu} d x^{\mu}$, and $F=d A+A^{2}$ with the external derivative $d$, and gauge transformation function $v=v^{a} T^{a}$ with the generators $T^{a}$ of $S U(N)$ gauge group. This is the so-called Stora-Zumino chain of the anomaly descent equations [四, B], 团], and the obtained consistent gauge anomaly is known to be the unique solution to the Wess-Zumino condition [ []]. The 5-th and 6-th dimensions in Refs. [0, 团] are introduced to describe a 2-parameter family of continuous interpolation from trivial gauge field to the reference gauge field and the gauge transformations. However, it is not clear whether these extra-dimensions have physical importance beyond a mathematical tool to derive the consistent anomaly.

In this work [ [8], we try to give more physical meaning of the extra-dimensions in a formulation of Weyl fermions. Namely, we consider the 5-th and 6-th directions as real coordinates, and put a massive Dirac fermion in 6-dimensional Euclidean space-time. Since this starting point is a massive and vector-like fermion system, we expect that a naive lattice discretization by the Wilson fermion is sufficient to non-perturbatively regularize it.

A Weyl fermion appears as a low-energy state localized on a 4-dimensional junction in the 6-dimensions. This situation is realized by putting a part of the 6-dimensional bulk space in a "topological" phase. More concretely, we introduce two kinds of domain-wall masses, perpendicular to each other, which give a gap to the 6-dimensional fermion in the bulk, but produces a gapless mode at the junction of the domain-walls. We may consider this set-up as a "doubly gapped topological insulator" which has a gapless mode at "the edge of the edges".

We find that the Stora-Zumino anomaly chain is naturally embedded in our 6-dimensional Dirac fermion determinant. The total determinant can be decomposed into 6-dimensional bulk, and 5, and 4-dimensional edge localized mode's contributions. The complex phase of the 6-dimensional bulk modes in the topological phase is nothing but the axial $U(1)$ anomaly. Since this $U(1)$ anomaly is a total divergence, its parity violation appear as a 5-dimensional surface contribution, which is compensated by the parity anomaly appearing in the phase of the 5-dimensional modes. The gauge transformation of the parity anomaly of the 5-dimensional modes is again a total divergence and its 4-dimensional surface contribution is then canceled by the gauge anomaly of the Weyl fermion at the 4-dimensional domain-wall junction.

Since the axial $U(1)$ symmetry is a global symmetry, its anomaly causes no theoretical problem in constructing a gauge theory in 6-dimensions. It is, however, an obstruction to give a 4dimensional local effective theory, since it contains the information of whole 6-dimensional gauge configuration even in the low-energy limit. If we are forced to consider such a theory as one in the 4-dimensional effective theory, it is no longer a local field theory. The anomaly-free sets of fermion 
flavors are the special exceptions where we can have a local 4-dimensional effective action. For the anomaly free fermion contents, the gauge and parity anomalies are canceled among flavors and never flow into the 6-dimensional bulk.

The two domain-walls must carry different quantum numbers. If they were constructed by the same operator, for example, the ordinary mass term in 6-dimensions, the resultant 4-dimensional mode would be not chiral but vector-like fermion having both of left- and right-handed modes. Therefore, it is a non-trivial issue how to choose the two domain-wall operators. We find several combinations of the two domain-walls producing the Weyl mode at the junction, and in fact, what we chose for the presentation at the conference turned out to be not appropriate for having non-local cancellation of the physical 4-dimensional phase of the determinant ${ }^{1}$. Here, we take another option: a combination of a simple mass term and an axial vector in 6-th direction to give the domain-wall profile.

Since the axial vector operator is invariant under the axial $U(1)$ rotation, our second domainwall: a kink structure of the axial vector operator, has nothing to do with the Stora-Zumino anomaly ladder. It is, however, not invariant under another anomalous symmetry: it flips the sign under reflection of 5-th coordinate. Since this symmetry is identical to the one yielding the parity anomaly in 5-dimensions, we call it $P^{\prime}$ symmetry (which is different from ordinary parity $P$ symmetry in 6dimension). To consider physical importance of $P^{\prime}$ symmetry, let us consider a single fundamental Dirac fermion in $S U(2)$ gauge theory. This fermion is known to have no axial $U(1)$ anomaly, which results in no perturbative gauge anomaly in 4-dimensions. However, this fermion can still have non-trivial zero-modes related to the mod-two index theorem reflecting the non-trivial homotopy group $\pi_{5}(S U(2))=\mathbb{Z}_{2}$. Now let us "assume" that this mod-two index is precisely counted by $P^{\prime}$ transformation. Then our second domain-wall should mediate its effects from the 5-dimensional bulk to the 5- and 4-dimensional edges, which may be regarded as the origin of global anomaly [Q]. In fact, we find a ladder of mod-two type indices:

$$
\pi_{5}(S U(2))=\mathbb{Z}_{2} \rightarrow \pi_{4}(S U(2))=\mathbb{Z}_{2} \quad \rightarrow \quad \text { complex phase of 4-d Weyl fermion. }
$$

Thus, our model contains an interesting possibility of the global anomaly as the consequence of the mod-two instantons in the 6-dimensions.

This work is strongly motivated by the recent work by Grabowska and Kaplan [एँ]], who proposed to construct the chiral gauge theory using the Yang-Mills gradient flow in the 5-th direction of their domain-wall fermion formulation, to maintain the exact 4-dimensional gauge invariance. We find that their idea can be easily extended to our 6-th dimension and we use the gradient flow to define the 4-dimensional path integration of the chiral gauge theory. In fact, taking the first domain-wall mass to infinity, our model converges to their 5-dimensional model. In this sense, our 6-dimensional proposal may be an extension of their work to incorporate the global anomaly. However, we need a further investigation to confirm this possibility since the use of the Yang-Mills gradient flow makes the role of global anomalies obscure: it seems not to allow non-trivial mod-two index in 6-dimensional bulk.

The rest of this article is organized as follows. In Sec. \, we define our model and show how a Weyl fermion appears at the domain-wall junction. Then in Sec. [ ] we show that the Stora-Zumino

\footnotetext{
${ }^{1}$ We thank D. B. Kaplan for pointing out this problem.
} 
anomaly chain is naturally embedded in our 6-dimensional Dirac fermion determinant. In Sec.⿴囗十 we discuss how the global anomalies appear in our model. The anomaly free condition in our model can be reduced to the cancellation of the complex phase in the 6-dimensional bulk, which comes from the global anomalous symmetries of the 6-dimensional Dirac fermions (Sec. ॠ). Finally we

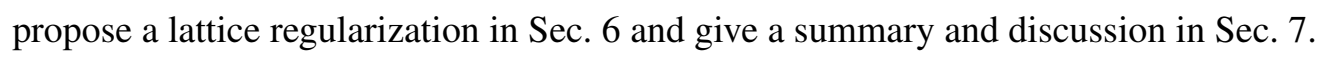

\section{Our 6-dimensional model}

In this work, we consider a 6-dimensional Euclidean space-time whose boundary condition for both of the fermions and bosons is periodic in every direction. Since we are interested in the 4-dimensional gauge theory, we simply take the 5-th and 6-th components of the gauge fields to be zero, i.e. $A_{5}(x)=A_{6}(x)=0$. For the other components, we require $A_{\mu}(\mu=1,2,3,4)$ to be symmetric under $x_{5} \rightarrow-x_{5}$ and $x_{6} \rightarrow-x_{6}$ (later we give more concrete form of $x_{5^{-}}$and $x_{6^{-}}$ dependences of $A_{\mu}$ by the Yang-Mills gradient flow).

Our 6-dimensional determinant of a Dirac fermion with the Pauli-Villars field is defined by

$$
\exp \left(-W_{2 \mathrm{DW}}\right) \equiv \operatorname{det}\left(\frac{D^{6 \mathrm{D}}+M \varepsilon\left(x_{6}\right)+i \mu \varepsilon\left(x_{5}\right) \gamma_{6} \gamma_{7}}{D^{6 \mathrm{D}}+M+i \mu \gamma_{6} \gamma_{7}}\right)
$$

where $D^{6 \mathrm{D}}$ denotes the 6-dimensional Dirac operator with a gauge group $S U\left(N_{c}\right), \varepsilon(x)=x /|x|$ denotes the sign function, and $\gamma_{i}$ are the $8 \times 8$ gamma matrices, with which the chiral operator is defined as $\gamma_{7}=i \prod_{i=1}^{6} \gamma_{i}$. Note that the two domain-walls parametrized by $M$ (we call the $M$ domain-wall) and $\mu$ ( $\mu$ domain-wall) intersect with each other at $x_{5}=x_{6}=0$, where a Weyl fermion appears. Since the fermion fields satisfy periodic boundary conditions, there also exist anti-domainwalls in the determinant. Although the anti-domain-walls do not appear in the expressions, we always assume that they are there, and will explicitly write them whenever it is necessary.

$M$ denotes the conventional Dirac fermion mass, whose domain-wall structure is known to produce massless fermions on it. As we will see below, this mass term is sensitive to the axial $U(1)$ transformation and is the origin of the perturbative gauge anomaly, which appears at the junction of the two domain-walls.

The $\mu$ term or the axial vector in the 6-th direction

$$
i \mu \bar{\psi} \gamma_{6} \gamma_{7} \psi
$$

on the other hand, is insensitive to the axial $U(1)$ rotation but flips its sign under a "parity" transformation $P^{\prime}$ or the reflection with respect to the $x_{5}$ axis:

$$
P^{\prime} \psi(x)=i \gamma_{5} R_{5} \psi(x), \quad \bar{\psi}(x) P^{\prime}=i R_{5} \bar{\psi}(x) \gamma_{5},
$$

where $R_{i}$ denotes the reflection of the $i$-th coordinate: $R_{i} f\left(x_{i}\right)=f\left(-x_{i}\right)$. Note that this parity is different from the conventional parity:

$$
P \psi(x)=\gamma_{1} \prod_{i \neq 1} R_{i} \psi(x), \quad \bar{\psi}(x) P=\prod_{i \neq 1} R_{i} \bar{\psi}(x) \gamma_{1},
$$

where we take $i=1$ to be the temporal direction. The main difference is that $P^{\prime 2}=-1$ while $P^{2}=1$. 
The $P^{\prime}$ symmetry has an anomaly [प], [2, [3]]. Since $\left\{D^{6 \mathrm{D}}, P^{\prime}\right\}=0$, every eigenvalue $i \lambda$ of $D^{6 D}$ has its pair - $i \lambda$, except for the zero modes. Therefore, under $P^{\prime}$, the massless fermion action is manifestly invariant, while the zero-mode's contribution to the fermion measure Jacobian is not, since $P^{\prime}$ flips its sign,

$$
D \bar{\psi}_{0} P^{\prime} D P^{\prime} \psi_{0}=-D \bar{\psi}_{0} D \psi_{0}
$$

Note that those from non-zero modes always cancel with their partners. Therefore, the $P^{\prime}$ transformation counts the number of zero-modes $\mathfrak{I}$. Here we would like to stress that $\mathfrak{I}$ can be different from the conventional topological charge, or the axial $U(1)$ index $\mathscr{P}$. An example is the $S U(2)$ theory where the $U(1)_{A}$ anomaly is exactly zero. Nevertheless, there exists the so-called mod-two index related to the homotopy group $\pi_{5}(S U(2))=\mathbb{Z}_{2}$. This homotopy group happens to be isomorphic to $\pi_{4}(S U(2))=\mathbb{Z}_{2}$, which describes the global anomaly [Q]. It is then natural to "assume" that the number of instantons are given by $\mathfrak{I}=\mathscr{P}+\mathscr{I}$, where $\mathscr{P}$ controls the perturbative gauge anomaly, while $\mathscr{I}$ is the origin of global anomalies.

As we have set $A_{5}(x)=A_{6}(x)=0$, we have $D^{6 \mathrm{D}}=D^{4 \mathrm{D}}+\gamma_{5} \partial_{5}+\gamma_{6} \partial_{6}$. Then we can easily find a solution of the Dirac equation

$$
\left(D^{6 \mathrm{D}}+M \varepsilon\left(x_{6}\right)+i \mu \varepsilon\left(x_{5}\right) \gamma_{6} \gamma_{7}\right) \psi(x)=0,
$$

localized at the domain-wall junction $x_{5}=x_{6}=0$ as

$$
\begin{aligned}
\psi(x) & =e^{-M\left|x_{6}\right|} e^{-\mu\left|x_{5}\right|} \phi(\bar{x}), \\
D^{4 \mathrm{D}} \phi(\bar{x}) & =0 \\
\gamma_{6} \phi(\bar{x}) & =\phi(\bar{x}), \\
i \gamma_{5} \gamma_{6} \gamma_{7} \phi(\bar{x}) & =\phi(\bar{x}),
\end{aligned}
$$

where $\bar{x}=\left(x_{1}, x_{2}, x_{3}, x_{4}\right)$ and we have assumed $M>0$ and $\mu>0$. Note that the latter two conditions Eqs. ([2.) and (D.JI) on $\phi(\bar{x})$ require the field to have a positive chirality (the opposite chirality can be realized by flipping the sign of $M$ and $\mu$ ). Namely, we have a single Weyl fermion localized at the domain-wall junction.

As a final remark of this section, we point out that the choice of the second domain-wall operator is not unique ${ }^{2}$. Here we have chosen the axial vector but other operators could reproduce the Weyl fermion mode at the junction as well. As mentioned in the introduction, some of them fail to reproduce the physical complex phase of the 4-dimensional effective theory. We need further investigation how to choose the appropriate combinations of the two domain-walls.

\section{Stora-Zumino anomaly ladder}

Now let us look into the structure of the anomaly ladder. This can be performed by decomposing the total Dirac fermion determinant into 6-, 5- and 4-dimensional mode's contributions. Details are shown in our paper [[]].

\footnotetext{
${ }^{2} \mathrm{~A}$ proposal of using the pseudoscalar operator for the second domain-wall was given by Neuberger in Ref. [(14]]) We also discuss other possibilities in our paper []].
} 
Let us begin with a simpler set-up taking $\mu=0$ :

$$
\operatorname{det}\left(\frac{D^{6 \mathrm{D}}+M \varepsilon\left(x_{6}\right)}{D^{6 \mathrm{D}}+M}\right) .
$$

We may regard this fermion determinant as a model for the 6-dimensional topological insulator, whose boundary is set at $x_{6}=0$ plane. This determinant is real and therefore, its complex phase can be written as $i \pi \Im$. Now we insert determinants with an intermediate cut-off $M_{2}$, to numerators and denominators (thus canceling each other) and take the $M \gg M_{2} \gg 0$ limit:

$$
\begin{aligned}
\operatorname{det}\left(\frac{D^{6 \mathrm{D}}+M \varepsilon\left(x_{6}\right)}{D^{6 \mathrm{D}}+M}\right)= & \operatorname{det}\left(\frac{D^{6 \mathrm{D}}+M \varepsilon\left(x_{6}\right)+i M_{2} \gamma_{6} \gamma_{7}}{D^{6 \mathrm{D}}+M}\right) \\
& \times \operatorname{det}\left(\frac{D^{6 \mathrm{D}}+M \varepsilon\left(x_{6}\right)}{D^{6 \mathrm{D}}+M \varepsilon\left(x_{6}\right)+i M_{2} \gamma_{6} \gamma_{7}}\right) .
\end{aligned}
$$

Here the first determinant corresponds to the 6-dimensional bulk contribution with an IR cut-off $M_{2}$ and the second one is the 5-dimensional edge mode's contribution whose UV cut-off is given by $M_{2}$.

We find by the Fujikawa's method, that the first determinant in Eq.(B.2) produces the axial $U(1)_{A}$ anomaly:

$$
\begin{aligned}
\operatorname{Im} \ln \operatorname{det}\left(\frac{D^{6 \mathrm{D}}+M \varepsilon\left(x_{6}\right)+i M_{2} \gamma_{6} \gamma_{7}}{D^{6 \mathrm{D}}+M}\right) & =\pi \int d^{6} x \frac{1-\varepsilon\left(x_{6}\right)}{2} \frac{1}{6(4 \pi)^{3}} \varepsilon^{\mu_{1} \cdots \mu_{6}} \operatorname{tr}\left[F_{\mu_{1} \mu_{2}} F_{\mu_{3} \mu_{4}} F_{\mu_{5} \mu_{6}}\right] \\
& =\pi \mathscr{P}_{x_{6}<0}^{6 D}+\pi C S
\end{aligned}
$$

where $\mathscr{P}_{x_{6}<0}^{6 D}$ counts the bulk instanton number in the region $x_{6}<0$, and $C S$ is the Chern-Simons term on the $M$ domain-wall,

$$
C S \equiv-\int_{x_{6}=0} d^{5} x \frac{2}{3(4 \pi)^{3}} \varepsilon^{\mu_{1} \cdots \mu_{5}} \operatorname{tr}\left[\frac{1}{2} A_{\mu_{1}} F_{\mu_{2} \mu_{3}} F_{\mu_{4} \mu_{5}}-\frac{i}{2} A_{\mu_{1}} A_{\mu_{2}} A_{\mu_{3}} F_{\mu_{4} \mu_{5}}-\frac{1}{5} A_{\mu_{1}} A_{\mu_{2}} A_{\mu_{3}} A_{\mu_{4}} A_{\mu_{5}}\right] .
$$

In the second determinant of Eq.(B.2), only the boundary localized mode satisfying $\gamma_{6} \psi=\psi$ and $\left(\gamma_{6} \partial_{6}+M \varepsilon\left(x_{6}\right)\right) \psi=0$, at the $M$ domain-wall can contribute. Rearranging the gamma-matrices, one obtains

$$
\lim _{M \rightarrow \infty} \operatorname{det}\left(\frac{D^{6 \mathrm{D}}+M \varepsilon\left(x_{6}\right)}{D^{6 \mathrm{D}}+M \varepsilon\left(x_{6}\right)+i M_{2} \gamma_{6} \gamma_{7}}\right)=\operatorname{det}\left(\frac{\bar{D}^{5 \mathrm{D}}}{\bar{D}^{5 \mathrm{D}}+M_{2}}\right),
$$

where the determinant in the RHS is taken in the reduced space of $4 \times 4$ gamma matrices $\bar{\gamma}_{i}$, and the corresponding Dirac operator is given by $\bar{D}^{5 \mathrm{D}}=\left.\sum_{i=1}^{5} \bar{\gamma}_{i}^{\prime} \nabla_{i}\right|_{x_{6}=0}$, where $\bar{\gamma}_{i}^{\prime}=i \bar{\gamma}_{5} \bar{\gamma}_{i}$.

It is known that the complex phase of the 5-dimensional massless Dirac fermion determinant in Eq. (B.5) is given by the so-called $\eta$-invariant [155, ㄸ6, ㅁ]], we have obtained a mathematical formula

$$
\mathfrak{I}=\mathscr{P}_{x_{6}<0}^{6 D}+C S-\frac{\eta^{5 D}}{2},
$$

known as the Atiyah-Patodi-Singer index theorem [18, [1, 20]]. 
Now let us take $\mu$ finite, and take the limit $M \gg \mu \gg 0$ :

$$
\begin{aligned}
\operatorname{det}\left(\frac{D^{6 \mathrm{D}}+M \varepsilon\left(x_{6}\right)+i \mu \varepsilon\left(x_{5}\right) \gamma_{6} \gamma_{7}}{D^{6 \mathrm{D}}+M+i \mu \gamma_{6} \gamma_{7}}\right) & =\operatorname{det}\left(\frac{D^{6 \mathrm{D}}+M \varepsilon\left(x_{6}\right)+i \mu \gamma_{6} \gamma_{7}}{D^{6 \mathrm{D}}+M+i \mu \gamma_{6} \gamma_{7}}\right) \\
& \times \operatorname{det}\left(\frac{D^{6 \mathrm{D}}+M \varepsilon\left(x_{6}\right)+i \mu \varepsilon\left(x_{5}\right) \gamma_{6} \gamma_{7}}{D^{6 \mathrm{D}}+M \varepsilon\left(x_{6}\right)+i \mu \gamma_{6} \gamma_{7}}\right) .
\end{aligned}
$$

The first determinant in Eq.(B.7) gives the same contribution as the one in Eq. (B.2), i.e. they yield the same contribution $\pi\left(\mathscr{P}_{x_{6}<0}^{6 D}+C S\right)$ to the phase of the determinant. This is consistent with the axial $U(1)_{A}$ insensitivity of the $\mu$ domain-wall.

The second determinant in Eq.(B.]) in the $M \rightarrow \infty$ limit, becomes

$$
\operatorname{det}\left(\frac{\bar{D}^{5 \mathrm{D}}+\mu \varepsilon\left(x_{5}\right)}{\bar{D}^{5 \mathrm{D}}+\mu}\right)
$$

which corresponds to the standard 5-dimensional domain-wall fermion determinant [ए]].

As shown in [ए]], or in our paper taking the anti-domain-wall effects more explicitly, the complex phase $-i \pi \eta^{5 \mathrm{D}} / 2$ (let us keep this notation given in the $\mu=0$ case) of the determinant Eq. (B.8) can be decomposed as

$$
-\frac{1}{2} \eta^{5 \mathrm{D}}=-C S^{\left(x_{5}<0\right)}-\mathscr{I}_{M \gg \mu}^{5 \mathrm{D}}-\frac{1}{2} \eta^{4 \mathrm{D}}+\frac{\phi^{\text {anom }}}{\pi},
$$

where we have another $C S$ term restricted to the $x_{5}<0$ region [ [2]]:

$$
\begin{aligned}
-\pi C S^{\left(x_{5}<0\right)} \equiv & \pi \int_{x_{6}=0} d^{5} x \frac{4}{3(4 \pi)^{3}} \frac{1-\varepsilon\left(x_{5}\right) \varepsilon\left(L_{5}-x_{5}\right)}{2} \varepsilon^{\mu_{1} \cdots \mu_{5}} \operatorname{tr}\left[\frac{1}{2} A_{\mu_{1}} F_{\mu_{2} \mu_{3}} F_{\mu_{4} \mu_{5}}\right. \\
& \left.-\frac{i}{2} A_{\mu_{1}} A_{\mu_{2}} A_{\mu_{3}} F_{\mu_{4} \mu_{5}}-\frac{1}{5} A_{\mu_{1}} A_{\mu_{2}} A_{\mu_{3}} A_{\mu_{4}} A_{\mu_{5}}\right]
\end{aligned}
$$

and $\mathscr{I}_{M \gg \mu}^{5 \mathrm{D}}$ counts the number of exotic instantons in 5-dimension. As 4-dimensional contributions from the Weyl fermion, we have a gauge invariant part denoted by $\eta^{4 \mathrm{D}}$, and the anomalous part $\phi^{\text {anom }}$ whose gauge symmetry breaking exactly cancels that in $C S^{\left(x_{5}<0\right)}$.

More explicitly, we obtain in the $\mu=\infty$ limit,

$$
\lim _{\mu \rightarrow \infty} \operatorname{det}\left(\frac{\bar{D}^{5 \mathrm{D}}+\mu \varepsilon\left(x_{5}\right)}{\bar{D}^{5 \mathrm{D}}+\mu}\right)=\exp \left(-i \pi C S^{\left(x_{5}<0\right)}-i \pi \mathscr{I}_{M \gg \mu}^{5 \mathrm{D}}\right) \times \lim _{\mu_{2} \rightarrow \infty} \operatorname{det} \frac{\mathscr{D}}{\mathscr{D}+\mu_{2}},
$$

where $\mathscr{D}$ is

$$
\mathscr{D}=P_{-}^{5} \bar{D}^{4 \mathrm{D}} P_{+}^{5}+P_{+}^{5} \bar{\partial}^{4 \mathrm{D}} P_{-}^{5},
$$

with $\bar{D}^{4 \mathrm{D}}=\left.\sum_{i=1}^{4} \bar{\gamma}_{i}^{\prime} \nabla_{i}\right|_{x_{6}=x_{5}=0}$ and $P_{ \pm}^{5}=\left(1 \pm \bar{\gamma}_{5}\right) / 2$. As will be shown later, we define the bulk gauge fields from the 4-dimensional gauge fields at the junction in such a way that the Dirac operator $\bar{\partial}^{4 \mathrm{D}}=\left.\sum_{i=1}^{4} \bar{\gamma}_{i}^{\prime} \nabla_{i}\right|_{x_{6}=0, x_{5}=L_{5}}$ becomes that for a (almost) free fermion, so that the negative chirality mode at $x_{5}=L_{5}$ is decoupled from the theory.

What we have obtained is the anomaly ladder

$$
\begin{aligned}
\phi^{\text {total }} / \pi & =\mathscr{P}_{x_{6}<0}^{6 D}+C S-\frac{\eta^{5 \mathrm{D}}}{2}, \\
\frac{1}{2} \eta^{5 \mathrm{D}} & =C S^{\left(x_{5}<0\right)}+\mathscr{I}_{M \gg \mu}^{5 \mathrm{D}}+\frac{1}{2} \eta^{4 \mathrm{D}}-\frac{\phi^{\text {anom }}}{\pi},
\end{aligned}
$$


where $\mathscr{P}_{x_{6}<0}^{6 D}$ denotes the 6-dimensional $U(1)_{A}$ anomaly, $C S$ and $C S^{\left(x_{5}<0\right)}$ represent the 5-dimensional parity anomaly, and $\phi^{\text {anom }}$ is the source of the consistent gauge anomaly. This result is consistent

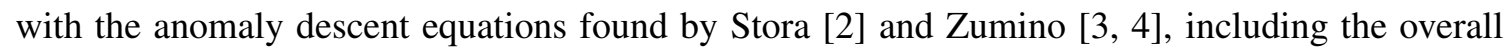
constant determined by Alvarez-Gaumé and Ginsparg [ [G] and Sumitani [ [ $]$ ].

\section{Global anomaly ladder?}

In order to trace the anomaly inflow via the $\mu$ domain-wall, let us take the limit $\mu \gg M \gg 0$. As will be shown below, the $\mu$ term is insensitive to the $U(1)_{A}$ rotation and there is no anomaly inflow related to the axial $U(1)_{A}$ anomaly, and therefore, the conventional perturbative gauge anomaly. A natural question is then to ask if there is any other anomaly flowing into the $\mu$ domain-wall or not. The answer is yes. As pointed out in Sec. \, we know an example of the exotic instantons and in $S U(2)$ gauge theory, which are insensitive to the $U(1)_{A}$ rotation. We assume here that these exotic instantons can be precisely detected by the $P^{\prime}$ anomaly. Then, we should have a non-trivial anomaly inflow through the $\mu$ domain-wall because it is not invariant under $P^{\prime}$ transformation.

Similarly to the previous section, our goal of this section is to decompose the complex phase of the determinant into three parts:

$$
\operatorname{Im} \ln \operatorname{det}\left(\frac{D^{6 \mathrm{D}}+M \varepsilon\left(x_{6}\right)+i \mu \varepsilon\left(x_{5}\right) \gamma_{6} \gamma_{7}}{D^{6 \mathrm{D}}+M+i \mu \gamma_{6} \gamma_{7}}\right)=\phi^{\mathrm{total}}=\phi^{6 \mathrm{D}}+\phi^{5 \mathrm{D}}+\phi^{4 \mathrm{D}},
$$

in the limit of $\mu \gg M \gg 0$.

First, let us decompose the determinant as

$$
\begin{aligned}
\operatorname{det}\left(\frac{D^{6 \mathrm{D}}+M \varepsilon\left(x_{6}\right)+i \mu \varepsilon\left(x_{5}\right) \gamma_{6} \gamma_{7}}{D^{6 \mathrm{D}}+M+i \mu \gamma_{6} \gamma_{7}}\right)= & \operatorname{det}\left(\frac{D^{6 \mathrm{D}}+i \mu \varepsilon\left(x_{5}\right) \gamma_{6} \gamma_{7}+M}{D^{6 \mathrm{D}}+i \mu \gamma_{6} \gamma_{7}+M}\right) \\
& \times \operatorname{det}\left(\frac{D^{6 \mathrm{D}}+i \mu \varepsilon\left(x_{5}\right) \gamma_{6} \gamma_{7}+M \varepsilon\left(x_{6}\right)}{D^{6 \mathrm{D}}+i \mu \varepsilon\left(x_{5}\right) \gamma_{6} \gamma_{7}+M}\right),
\end{aligned}
$$

where the first part corresponds to the 6-dimensional bulk contribution and the second is the one from 5- and 4-dimensional boundary.

Note again that unlike the $M$ domain-wall, the first determinant of Eq. (4.2) does not produce the axial $U(1)$ anomaly. Due to the explicit violation of the $S O(6)$ Lorentz symmetry by the axial vector background, the phase $\phi^{6 \mathrm{D}}$ of the second determinant can be expanded in an $S O(5)$ invariant series of $1 / \mu$, except for the non-perturbative zero mode's contribution $\pi \mathscr{I}_{x_{5}<0}^{6 \mathrm{D}}$, which is located in the region $x_{5}<0$. More explicitly, we obtain by one-loop computation

$$
\phi^{6 \mathrm{D}}=\pi \mathscr{I}_{x_{5}<0}^{6 \mathrm{D}}+\mu \phi^{(1)}+\mathscr{O}(1 / \mu),
$$

where, the leading order contribution has a form of the Chern-Simons term

$$
\phi^{(1)}=c_{0} \pi \int d^{6} x \frac{4}{3(4 \pi)^{3}} \frac{1-\varepsilon\left(x_{5}\right)}{2} \varepsilon^{i_{1} \cdots i_{5}} \operatorname{tr}\left[\frac{1}{2} A_{i_{1}} F_{i_{2} i_{3}} F_{i_{4} i_{5}}-\frac{i}{2} A_{i_{1}} A_{i_{2}} A_{i_{3}} F_{i_{4} i_{5}}-\frac{1}{5} A_{i_{1}} A_{i_{2}} A_{i_{3}} A_{i_{4}} A_{i_{5}}\right],
$$

where $c_{0}$ is a constant. Since $\phi^{(1)}$ cancels among the anomaly free fermion contents, the only non-trivial phase is given by $\pi \mathscr{I}_{x_{5}<0}^{6 \mathrm{D}}$ when the perturbative gauge anomaly is absent. 
The second determinant of Eq. (4.2) in the $\mu \rightarrow \infty$ limit converges to ${ }^{3}$

$$
\begin{aligned}
& \operatorname{det}\left(P_{+}\left(\hat{D}^{5 \mathrm{D}}+M\right)^{-1}\left(\hat{D}^{5 \mathrm{D}}+M \varepsilon\left(x_{6}\right)\right) P_{+}+P_{-}\right) \\
& \times \operatorname{det}\left(P_{+}\left(\hat{D}^{5 \mathrm{D}}-M\right)^{-1}\left(\hat{D}^{5 \mathrm{D}}-M \varepsilon\left(x_{6}\right)\right) P_{+}+P_{-}\right),
\end{aligned}
$$

where $\hat{D}^{5 \mathrm{D}}=\left.\left(\sum_{i=1}^{4} \bar{\gamma}_{i} \nabla_{i}+\bar{\gamma}_{5} \partial_{6}\right)\right|_{x_{5}=0}$, and $P_{ \pm} \equiv\left(1 \pm \bar{\gamma}_{5}\right) / 2$ are Hermitian projection operators. Unlike the case in the previous section, what we obtain here on the $\mu$ domain-wall is not a single Dirac fermion but two (4-component) Dirac fermions having Pauli-Villars masses $\pm M$ with opposite signs, that are constrained to have the positive eigenvalue of the gamma matrix $\bar{\gamma}_{5}$. This expression is almost real, except for the domain-wall $x_{6}=0$, since the complex phase comes from the non-commutativity of $\hat{D}^{5 \mathrm{D}}$ and $M \varepsilon\left(x_{6}\right)$, which is proportional to $\delta\left(x_{6}\right)$. We can thus express the phases of the 5- and 4-dimensional contributions as

$$
\begin{aligned}
\phi^{5 \mathrm{D}} & =\pi \mathscr{I}_{x_{6}<0}^{5 \mathrm{D}}, \\
\phi^{4 \mathrm{D}} & =-\frac{\pi}{2} \eta^{4 \mathrm{D}},
\end{aligned}
$$

assuming that the $M \gg \mu \gg 0$ and $\mu \gg M \gg 0$ limits commute to obtain the same gauge invariant part of $\phi^{4 \mathrm{D}}$ as in the previous section. It is natural to assume that $\mathscr{I}_{x_{6}<0}^{5 \mathrm{D}}$ gives the mod-two instanton found by Witten [Q] which describes the global gauge anomaly.

Note in Eq. (4.5), only the Weyl fermion with positive $M$ appears in the low-energy limit, while the other leaves a non-local phase, which cannot be described by any local action. We consider the latter non-local phase as the contribution of the Chern-Simons, which automatically cancels the perturbative gauge anomalies.

To summarize this section, we have confirmed the anomaly inflow via $\mu$ domain-wall,

$$
\phi^{\text {total }}=\pi \mathscr{I}_{x_{5}<0}^{6 \mathrm{D}}+\pi \mathscr{I}_{x_{6}<0}^{5 \mathrm{D}}-\frac{\pi}{2} \eta^{4 \mathrm{D}}+\mu \phi^{(1)}+\mathscr{O}(1 / \mu)
$$

where the mod-two type indices $\mathscr{I}_{x_{5}<0}^{6 \mathrm{D}}$ and $\mathscr{I}_{x_{6}<0}^{5 \mathrm{D}}$ are balanced in a non-trivial way with the 4dimensional phase $\eta^{4 \mathrm{D}}$ (even when the perturbative gauge anomaly and $\phi^{(1)}$ are absent).

\section{Anomaly free condition}

In the previous two sections, we have traced two different anomaly inflows taking $M \gg \mu \gg 0$ and $\mu \gg M \gg 0$ limits. At finite $M$ and $\mu$, the situation can be more complicated but the non-trivial cancellation of anomalies among different dimensions should be maintained to keep the gauge invariance of the total theory. In the end, a single Weyl fermion always appears at the junction of the two domain-walls.

When a small gauge transformation is performed at the 4-dimensional junction, the gauge current flows through the $M$ domain-wall, but never flows into the $\mu$ domain-wall, since there is no CS term which can absorb the gauge non-invariance. Instead, a large gauge transformation can create exotic instantons on the $\mu$ domain-wall and flip the sign of the partition function. Thus, we confirm that the perturbative anomaly inflow, which naturally exhibits the Stora-Zumino anomaly

\footnotetext{
${ }^{3} \mathrm{As} \hat{D}^{5 \mathrm{D}}$ and $P_{+}$do not commute with $M \varepsilon\left(x_{6}\right)$, we make the order of the matrix operations explicit.
} 
descent equations, is mediated by the $M$ domain-wall, while the inflow of the global anomaly goes through the $\mu$ domain-wall (see Fig. $\mathbb{W}$ ).

Now the perturbative and global gauge anomalies are viewed as these missing gauge current inflows into the 5- and 6-dimensional bulk. If these currents happen to cancel among different flavors (of the 6-dimensional Dirac fermion), the heavy bulk mode fermions can be decoupled from the low-energy theory, leaving the 4-dimensional effective theory of Weyl fermions. Thus, the anomaly free condition is to require the cancellation of the $U(1)_{A}$ anomaly and the $P^{\prime}$ anomaly in 6-dimensional Dirac fermions. This condition is equivalent to the cancellation of the phase of the bulk fermion determinant mod $2 \pi \times$ integer, which agrees with the recent discussion by Witten [ㄹ3]. Note that the 4-dimensional edge modes at the domain-wall junction are still allowed to have their own complex phase.

The cancellation of the $U(1)_{A}$ anomaly is guaranteed if

$$
\sum_{L} \operatorname{tr} T_{L}^{a}\left\{T_{L}^{b}, T_{L}^{c}\right\}-\sum_{R} \operatorname{tr} T_{R}^{a}\left\{T_{R}^{b}, T_{R}^{c}\right\}=0,
$$

where $T_{L / R}$ denote the gauge group generators in $L / R$ representation of the corresponding left/right handed fermions. This condition assures the cancellation of the $U(1)_{A}$ anomaly, as well as the CS term on the $M$ domain-wall, so that the gauge current never flows out of the 4-dimensional junction.

The cancellation of the global anomalies is more non-trivial, as discussed in [22], 223]. The global anomaly should be absent not only on a simple manifold like $S^{4}$ or $S^{5}$ but also on any compact manifold. Our set-up on the 6-dimensional torus having domain-wall junctions of 4dimensional torus, is already such a non-trivial example.

Here we take the most conservative condition:

number fermions in the fundamental representation = even,

after the irreducible decomposition. Since the phase from the the exotic index $\mathscr{I}$ is always multiplied by $2 \pi \times$ integer, the global anomaly effect is absent in the 5 - and 6-dimensional bulkmodes. Note that the standard model of particle physics satisfies the above condition if we identify $e / 6$ as a unit charge of the hyper-charge.

\section{Lattice regularization}

Since our 6-dimensional formulation is based on a massive Dirac fermion, it is natural to assume that we can non-perturbatively regularize it on a lattice using the Wilson Dirac operator.

First let us put our lattice in a 6-dimensional finite box. In particular, we take the 5-th and 6-th coordinates in the ranges $-L_{5}<x_{5} \leq L_{5}$ and $-L_{6}<x_{6} \leq L_{6}$ and assume the periodic boundary condition of the Dirac fields in every direction. Because of the boundary condition, we need (at least) one $M$ anti-domain-wall at $x_{6}=L_{6}\left(=-L_{6}\right)$ and one $\mu$ anti-domain-wall at $x_{5}=L_{5}\left(=-L_{5}\right)$. Then we have 4 domain-wall junctions. Two Weyl fermion modes with positive chirality appear at $\left(x_{5}, x_{6}\right)=(0,0)$ and $\left(0, L_{6}\right)$, while those with negative chirality are localized at $\left(x_{5}, x_{6}\right)=\left(L_{5}, 0\right)$ and $\left(L_{5}, L_{6}\right)$.

Among these 4 junctions, only the one at $\left(x_{5}, x_{6}\right)=(0,0)$ is needed to formulate the Weyl fermion in 4-dimensions. Therefore, the other massless fermions at other three junctions have to 


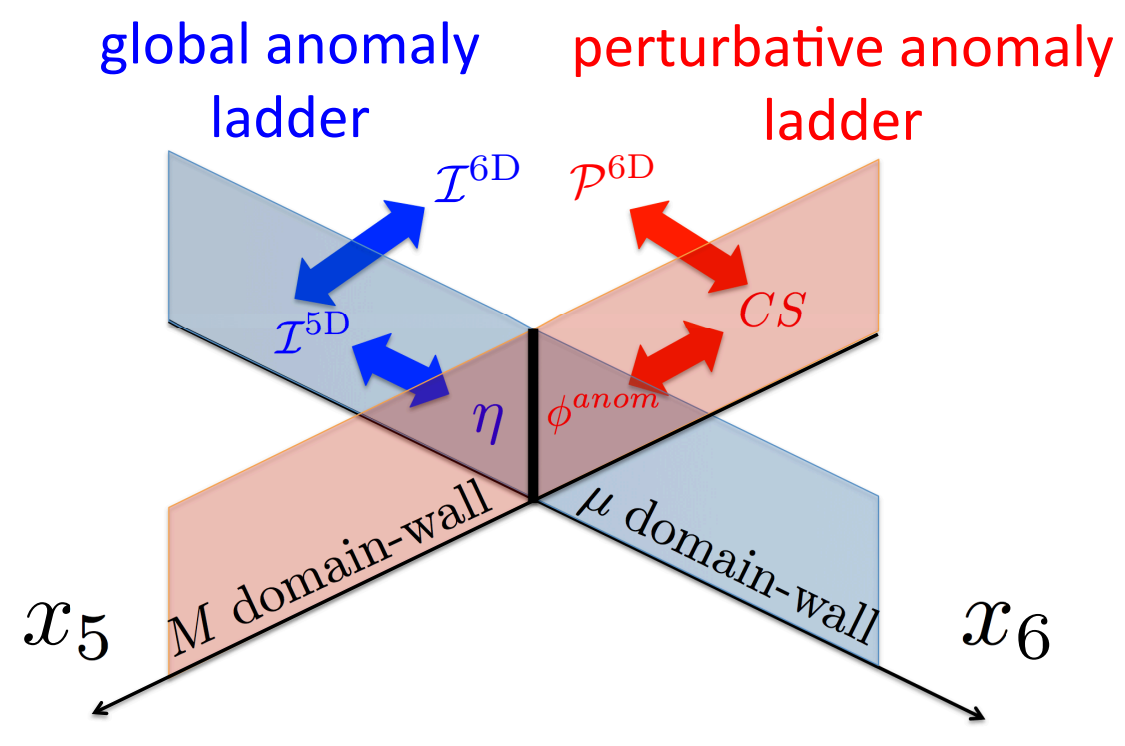

Figure 1: The anomaly inflows through the two domain-walls. The $M$ domain-wall at $x_{6}=0$ mediates the perturbative anomaly inflow (red arrows), which exhibits the Stora-Zumino descent equations. The $\mu$ domain-wall at $x_{5}=0$ mediates the inflow of the global anomaly (blue).

be decoupled from the total theory. To achieve this, we follow the idea in [ए]] using the Yang-Mills gradient flow in the fifth and sixth directions. The gradient flow exponentially weaken the gauge fields with the flow time so that the Weyl fermions at $x_{5}=L_{5}$ and $x_{6}=L_{6}$ are decoupled from the gauge fields ${ }^{4}$. As flowed fields transform in the same way as the original fields under the gauge transformation, we can maintain the 4-dimensional gauge invariance of the total theory.

More concretely, we pick up a set of link variables $\left\{U_{\mu}(\bar{x})\right\}(\mu=1, \cdots 4)$ on the 4-dimensional junction at $\left(x_{5}, x_{6}\right)=(0,0)$. Then we solve the lattice version of the Yang-Mills gradient flow equation,

$$
\frac{\partial}{\partial t} U_{\mu}^{t}(\bar{x})=-\left\{\partial_{x, \mu} S_{G}\left(U^{t}\right)\right\} U_{\mu}^{t}(\bar{x}),
$$

using $U_{\mu}^{0}(\bar{x})=U_{\mu}(\bar{x})$ as the initial condition, where $\partial_{x, \mu} S_{G}\left(U^{t}\right)$ denotes the Lie derivative of the gauge action $S_{G}\left(U^{t}\right)$ with respect to $U_{\mu}^{t}(\bar{x})$, to define

$$
U_{\mu}\left(\bar{x}, x_{5}, x_{6}\right)=U_{\mu}^{\left|x_{5}\right|+\left|x_{6}\right|}(\bar{x}) .
$$

Here we always set $U_{5}=U_{6}=$ unity. Note that the resulting link variables $U_{\mu}\left(\bar{x}, x_{5}, x_{6}\right)$ are symmetric under $x_{5} \rightarrow-x_{5}$ and $x_{6} \rightarrow-x_{6}$.

\footnotetext{
${ }^{4}$ Recently it was proved that this is true except for those coming from the non-trivial topologies. See Refs. [24, [25, [26]
} 
Finally we "define" the 4-dimensional path integral of anomaly free theory with Weyl fermions. Together with the gauge part of the action $S_{G}\left(\left\{U_{\mu}(\bar{x})\right\}\right)$, we define

$$
\int D U_{\mu}(\bar{x}) e^{-S_{G}\left(\left\{U_{\mu}(\bar{x})\right\}\right)} \prod_{i} \exp \left[-W_{\text {lat }}^{i}\left(\left\{U_{\mu}(\bar{x})\right\}\right)\right],
$$

where

$$
\begin{gathered}
\exp \left[-W_{\text {lat }}^{i}\left(\left\{U_{\mu}(\bar{x})\right\}\right)\right]= \\
\operatorname{det}\left(\frac{D_{W}^{6 \mathrm{D} R_{i}}+M_{i} \varepsilon\left(x_{6}-a / 2\right) \varepsilon\left(L_{6}-x_{6}-a / 2\right)+i \mu_{i} \varepsilon\left(x_{5}-a / 2\right) \varepsilon\left(L_{5}-x_{5}-a / 2\right) \gamma_{6} \gamma_{7}}{D_{W}^{6 \mathrm{D} R_{i}}+M_{i}+i \mu_{i} \gamma_{6} \gamma_{7}}\right),
\end{gathered}
$$

where $D_{W}^{6 \mathrm{D} R_{i}}$ denotes the Wilson Dirac operator in the $R_{i}$ representation of the gauge group, and $M_{i}$ and $\mu_{i}$ are chosen to be positive/negative for positive/negative chiral modes. Note that the Wilson term has to have an opposite sign to $M_{i}$ and $\mu_{i}$. These mass parameters are to be of the order of the lattice cut-off $1 / a$. However, to avoid contamination from the doubler modes, $M_{i}$ and $\mu_{i}$ should satisfy some upper bounds. We always assume that the set of fermion flavors satisfy the anomaly free conditions Eqs. (5.]) and (5.2). Our set-up is presented in Fig. ㅁ.

\section{Summary and discussion}

We have proposed a regularization of the chiral gauge theories in 4-dimensions, using massive 6-dimensional Dirac fermions. Using the two different kinds of domain-walls, we have succeeded in localizing a single Weyl fermion at the junction of the domain-walls. One domain-wall is made giving a kink mass in the 6-th direction to the fermions, while another domain-wall is made by giving a kink structure in the 5-th direction to a background operator which is insensitive to the $U(1)_{A}$ rotation. Our set-up can be viewed as a "doubly" gapped topological insulator, whose 4dimensional edge modes become massless.

The domain-wall of the conventional mass term mediates the perturbative anomaly inflow and naturally exhibits the chain of the 6-dimensional $U(1)_{A}, 5$-dimensional parity, and 4-dimensional

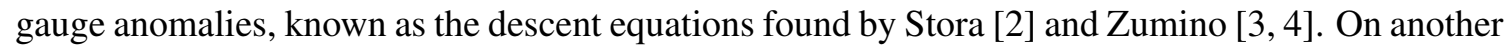
domain-wall, the fermions are forced to form (almost) a real representation and only mediates the mod-two type anomaly, which we have assumed to be the source of the global anomalies.

The anomaly free condition of the target 4-dimensional gauge theory is translated to the cancellation of the axial $U(1)$ and $P^{\prime}$ anomalies for a set of 6-dimensional Dirac fermions. This condition removes the total complex phase from the bulk part of the fermion determinant, while the 4-dimensional edge modes can have their own phase. Using the Yang-Mills gradient flow in the 5-th and 6-th directions, we can control the remnant of the gauge non-invariance due to the finite cut-offs, and decouple the Weyl fermions at the junctions of anti-domain-walls. As our formulation is nothing but a massive vector-like theory, we expect that a non-perturbative regularization on a lattice is possible, using standard Wilson Dirac fermions.

There are still a lot of open issues to be investigated. There is an arbitrariness in the choice of the $\mu$ domain-wall operator, to realize a single Weyl fermion at the domain-wall junction. It is 


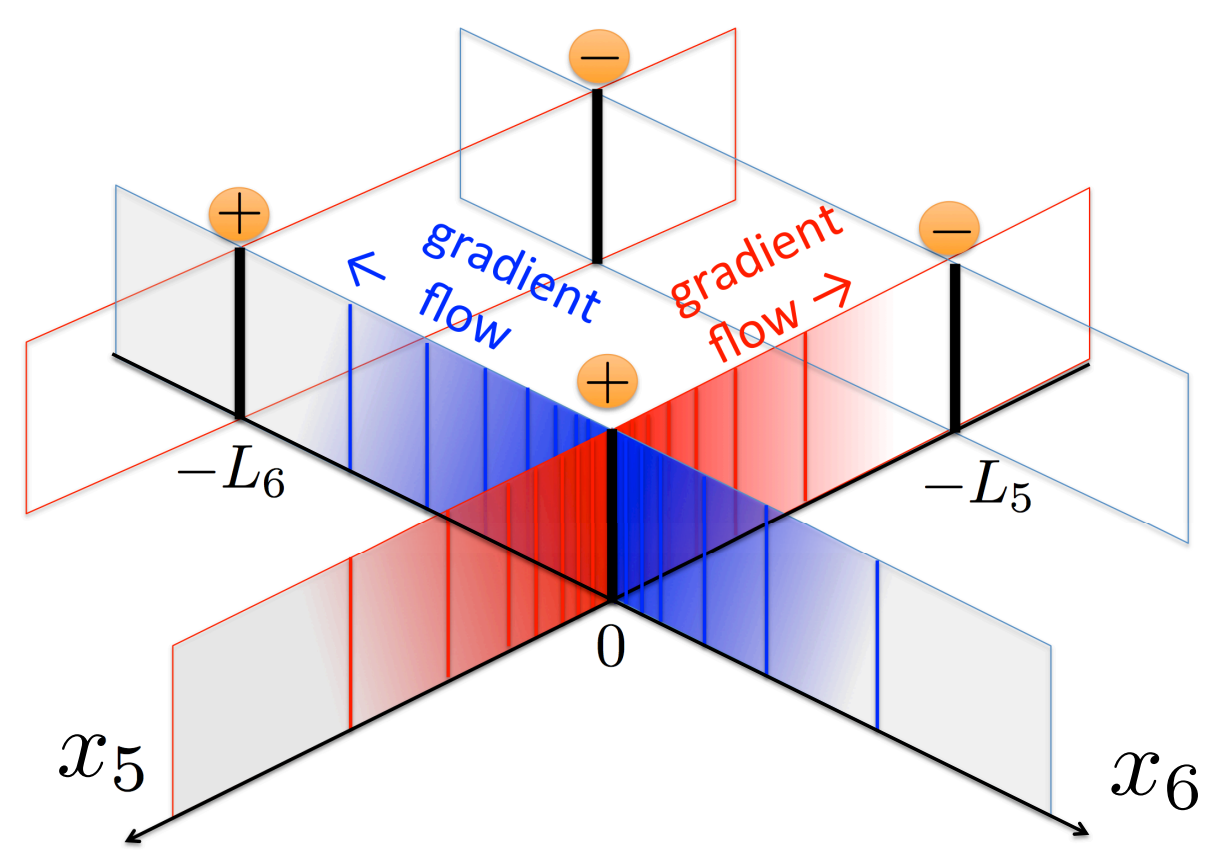

Figure 2: Schematic view of our 6-dimensional finite lattice. The \pm symbols show the Weyl modes with positive and negative chiralities, localized at each of the four domain-wall junctions. The case with $M>0$ and $\mu>0$ is shown. Our target Weyl fermion with positive chirality is localized at the origin, while other three Weyl fermions are decoupled from the gauge fields by the gradient flow.

also unclear if the $\mu$ domain-wall and associated $P^{\prime}$ anomaly necessarily and sufficiently classify the global anomalies.

It is an interesting question if our formulation can be extended to a model with physical extra dimensions also in the gauge sector. Such a direction may be linked to studies of higher dimensional beyond the standard models. Our formulation suggests that there is a possibility of doubly gapped topological insulators in four-dimensions, having a conducting mode on two-dimensional edges, which may be realized in condensed matter systems. Finally, it would be great if we can incorporate the Higgs field to our 6-dimensional lattice and give a non-perturbative definition of the standard model, which is also an interesting subject for further study.

We thank S. Aoki, D. Grabowska, K. Hashimoto, D. B. Kaplan, Y. Kikukawa, H. Suzuki, and S. Yamaguchi for useful discussions. This work is supported in part by the Grand-in-Aid of the Japanese Ministry of Education Nos. 25800147, 26247043 (H.F.), No. 26400248(T.O.), and No. 15J01081 (R.Y.).

\section{References}

[1] S. Weinberg, "The Quantum Theory of Fields II," Cambridge University Press (1995)

[2] R. Stora, LAPP-TH-94, C83-09-01. 
[3] B. Zumino, In *Treiman, S.b. ( Ed.) Et Al.: Current Algebra and Anomalies*, 361-391 and Lawrence Berkeley Lab. - LBL-16747 (83,REC.OCT.) 46p

[4] B. Zumino, Y. S. Wu and A. Zee, Nucl. Phys. B 239, 477 (1984). doi:10.1016/0550-3213(84)90259-1

[5] J. Wess and B. Zumino, Phys. Lett. 37B, 95 (1971). doi:10.1016/0370-2693(71)90582-X

[6] L. Alvarez-Gaume and P. H. Ginsparg, Nucl. Phys. B 243, 449 (1984). doi:10.1016/0550-3213(84)90487-5

[7] T. Sumitani, J. Phys. A 17, L811 (1984). doi:10.1088/0305-4470/17/14/016

[8] H. Fukaya, T. Onogi, S. Yamamoto and R. Yamamura, arXiv:1607.06174 [hep-th].

[9] E. Witten, Phys. Lett. B 117, 324 (1982). doi:10.1016/0370-2693(82)90728-6

[10] D. M. Grabowska and D. B. Kaplan, Phys. Rev. Lett. 116, no. 21, 211602 (2016) doi:10.1103/PhysRevLett.116.211602 [arXiv:1511.03649 [hep-lat]].

[11] A. N. Redlich, Phys. Rev. D 29, 2366 (1984). doi:10.1103/PhysRevD.29.2366

[12] A. J. Niemi and G. W. Semenoff, Phys. Rev. Lett. 51, 2077 (1983). doi:10.1103/PhysRevLett.51.2077

[13] L. Alvarez-Gaume, S. Della Pietra and G. W. Moore, Annals Phys. 163, 288 (1985). doi:10.1016/0003-4916(85)90383-5

[14] H. Neuberger, hep-lat/0303009.

[15] L. Alvarez-Gaume, S. Della Pietra and V. Della Pietra, Phys. Lett. B 166, 177 (1986). doi:10.1016/0370-2693(86)91373-0

[16] S. Della Pietra, V. Della Pietra and L. Alvarez-Gaume, Commun. Math. Phys. 109, 691 (1987). doi:10.1007/BF01208963

[17] D. B. Kaplan and M. Schmaltz, Phys. Lett. B 368, 44 (1996) doi:10.1016/0370-2693(95)01485-3 [hep-th/9510197].

[18] M. F. Atiyah, V. K. Patodi and I. M. Singer, Math. Proc. Cambridge Phil. Soc. 77, 43 (1975). doi:10.1017/S0305004100049410

[19] M. F. Atiyah, V. K. Patodi and I. M. Singer, Math. Proc. Cambridge Phil. Soc. 78, 405 (1976). doi:10.1017/S0305004100051872

[20] M. F. Atiyah, V. K. Patodi and I. M. Singer, Math. Proc. Cambridge Phil. Soc. 79, 71 (1976). doi:10.1017/S0305004100052105

[21] C. G. Callan, Jr. and J. A. Harvey, Nucl. Phys. B 250, 427 (1985). doi:10.1016/0550-3213(85)90489-4

[22] X. z. Dai and D. S. Freed, J. Math. Phys. 35, 5155 (1994) Erratum: [J. Math. Phys. 42, 2343 (2001)] doi:10.1063/1.530747 [hep-th/9405012].

[23] E. Witten, Rev. Mod. Phys. 88, no. 3, 035001 (2016) doi:10.1103/RevModPhys.88.035001, 10.1103/RevModPhys.88.35001 [arXiv:1508.04715 [cond-mat.mes-hall]].

[24] K. i. Okumura and H. Suzuki, arXiv:1608.02217 [hep-lat].

[25] H. Makino and O. Morikawa, arXiv:1609.08376 [hep-lat].

[26] D. M. Grabowska and D. B. Kaplan, arXiv:1610.02151 [hep-lat]. 